\title{
Construyendo sobre arcoíris: apoyando la participación de niños, niñas y adolescentes en la configuración de respuestas al COVID-19 ${ }^{1}$
}

\author{
Lucía del Moral-Espín²; Cath Larkins ${ }^{3}$
}

La participación de los niños, niñas y adolescentes en la toma de decisiones no es un lujo, es una medida de protección (Warrington y Larkins, 2019). Ellos y ellas han demostrado repetidamente que, ante situaciones de catástrofe como terremotos, inundaciones o incendios, pueden hacer $\mathrm{y}$, de hecho, hacen valiosísimas contribuciones para dar respuesta a los desastres y favorecer la reducción de los riesgos ${ }^{4}$ (en particular en situaciones vulnerables). En el contexto actual de crisis generada por la COVID-19, el Comité de los Derechos del Niño, en una declaración publicada a principios de abril 2020, no solo advertía del grave impacto físico, emocional y psicológico de la pandemia y de las medidas adoptadas frente a ella en los sectores más jóvenes de la población, sino que pedía a los Estados que tomasen medidas, subrayando que las opiniones de los niños, niñas y adolescentes deberían ser tenidas en cuenta en la configuración y desarrollo de las políticas de respuesta. "Construyendo sobre arcoíris" presenta las conclusiones de la primera fase (13-20 abril de 2020) de una investigación, basada en una encuesta online, sobre el impacto la COVID-19 en las vidas de los niños y sobre la configuración de las medidas ante la pandemia en el contexto europeo 5 . Las personas informantes, 95 profesionales de 16 sectores y áreas de conocimiento diferentes (academia, medio de comunicación, defensa de derechos de los niños, protección de la infancia, educación, artes participativas, psicología, salud pública, pedagogía social, trabajo social y comunitario entre otros), fueron contactadas a través de redes de participación y derechos de la infancia en 20 países europeos, España entre ellos. ${ }^{6}$ A partir de los enlaces proporcionados en las respuestas a los formularios, se tuvo acceso a otras fuentes generadas directamente por niños, niñas o adolescentes. Así mismo, se desarrolló una discusión online focalizada con personas expertas de 16 países europeos para verificar los datos recogidos y recabar información adicional. De manera general, los datos dibujan en siguiente contexto generado, más bien acentuado por la COVID-19:

- En todos los países, niños, niñas y adolescentes se han visto afectados por problemas en materia de salud, barreras a la comunicación y participación, escasez de información y dependencia digital. Además, en muchos contextos, hay dificultades adicionales para dar cobertura a las necesidades básicas, garantizar el acceso a la educación, a los servicios sociales o de salud mental, así como para salvaguardar de la exposición a situaciones de violencia, de la reducción de los ingresos del hogar y del aumento de distintas formas de discriminación.

- Estas condiciones y desafíos se intensifican cuando se trata de niños, niñas o adolescentes en el sistema de protección, con discapacidad, de origen migrante, de etnia gitana, que viven situación de pobreza o con exposición a la explotación sexual, tráfico y violencia.

- A nivel nacional, local y organizacional se han introducido medidas para tratar de abordar estos desafíos y mitigar los riesgos. Sin embargo, su diseño e implementación no han contado con los y las más jóvenes.

\footnotetext{
Este texto consiste en un resumen y traducción al castellano del informe "Building on Rainbows Supporting Children's Participation in Shaping Responses to COVID-19. Rapid Evidence Report” dirigido por Cath Larkins con la colaboración de Daniel Stoecklin, Rositsa Milkova, Lucia Del Moral-Espín, Anne Crowley, Maggie Mort, Lucy Easthope, Mieke Schurmann, Deborah Crook and Natália Fernandes. Editado por The Centre for Children and Youn People's Participation. Preston, accesible en https://www.observatoriodelainfancia.es/oia/esp/documentos_ficha.aspx?id=7071

Universidad de Cádiz.

E-mail: lucia.delmoral@uca.es

University of Central Lancashire (Reino Unido).

E-mail: clarkins@uclan.ac.uk

4 Ver conclusiones del proyecto CUIDAR y el informe Words into Action guidelines: Engaging children and youth in disaster risk reduction and resilience building de la Oficina de Naciones Unides para la Reducción de Riesgo de Desastres (UNDRR).

5 Durante el mes de mayo 2020 el formulario sigue abierto, las nuevas respuestas recogidas serán analizadas y los resultados publicados en un segundo informe.

6 Los otros países serían: Albania, Alemania, Bélgica, Bulgaria, Croacia, Dinamarca, Francia, Irlanda, Italia, Kosovo, Macedonia del Norte, Noruega, Polonia, Portugal, Rumania, Serbia, Suiza, los Países Bajos y el Reino Unido.
} 
La participación implica un proceso continuo, si bien nunca lineal, de planificación, conexión con las personas, identificación de problemas, estudio de opiniones, adopción de medidas, seguimiento y revisión de las acciones. Durante la pandemia, tal y como han mostrado diversos estudios ${ }^{7}$, los niños, niñas y adolescentes han contribuido activamente al funcionamiento y bienestar de los hogares, han creado y compartido información para promover la salud y seguridad, cuidado, proporcionado ayuda y apoyo a otros miembros de sus familias, personas amigas y vecinas, participado en distintas formas de trabajo y, en ocasiones, fomentando una mejora de los servicios. Sin embargo, el $70 \%$ de las personas de nuestra red de informantes no pudo identificar ni una sola iniciativa de participación infantil relacionada con la COVID-19, a nivel local, regional, nacional o internacional. De las reportadas, ninguna hasta el momento, había dado lugar al desarrollo de medidas gubernamentales más allá de campañas, actos de comunicación e información dirigida específicamente a la infancia y adolescencia.

\section{Ejemplos y logros de participación infantil relacionados con COVID-19}

La gran mayoría del 30\% de informantes que declaró conocer alguna práctica de participación, señaló actividades iniciadas por sus propias organizaciones. Se recogieron referencias a acciones en desarrollo o necesarias en 11 países. Las acciones más veces mencionadas fueron las de contacto con niños, niñas o adolescentes, seguidas de estudios de opinión, identificación de cuestiones de interés, procesos de participación que se encuentran en sus primeras etapas de preparación y planificación, adopción de medidas y, como necesidad, pero aún no plenamente desarrolladas, seguimiento de medidas y revisión e intercambio de experiencias y prácticas.

Paralelamente, los niños, niñas o adolescentes han venido desarrollando y participando en actividades autopromovidas y autodirigidas orientadas a la colaboración y generación de cambios, como campañas en Facebook y en Instagram, sobre las representaciones de la infancia y juventud en relación con la COVID-19 en los medios de comunicación. Aunque algunos formularios hacían referencia a estos procesos, ninguno ofreció información detallada al respecto.

En lo relativo a la recepción, diseño e intercambio de información, estudios previos (como el antes señalado CUIDAR) han demostrado la importancia de no eludir el hablar de las crisis de salud pública con los niños, niñas y adolescentes, sino hacerlo de manera apropiada a su edad. En este sentido, según la información recogida, el personal técnico y profesional ha venido desarrollando actividades on-line y off-line para conectar con ellos y ellas, tanto a nivel individual como grupal, y distintos grupos de participación siguen reuniéndose virtualmente y creando actividades. En algunos países (como España a nivel estatal y también en alguna Autonomía), se habrían abierto espacios específicos en televisión y/o redes sociales donde niños, niñas y adolescentes han planteado preguntas a los/as responsables políticos y sanitarios.

En cuanto a la recogida de los puntos de vista particulares o colectivos, se sigue teniendo algún contacto directo y se han desarrollado distintas encuestas y estudios cualitativos. En cuanto a las acciones vinculadas a la planificación y configuración de servicios, se reportan ejercicios de aprendizaje mutuo para recoger y reconocer los conocimientos y perspectivas de los niños sobre el riesgo y el peligro (por ejemplo, con profesionales de protección civil en Cataluña). Niños, niñas y adolescentes han participado en el diseño y la experimentación de soluciones relacionadas con el bienestar, las exclusiones digitales y en el diseño propuestas de proyectos para presentar a convocatorias de financiación. Así mismo, se informa de que ha habido cierta colaboración con Comisionados de Infancia y/o Defensorías de derechos de la infancia y se han desarrollado campañas y acciones vinculadas a los derechos de los niños, al cuidado alternativo y la educación inclusiva.

Para facilitar la participación de niños, niñas y adolescentes, las personas informantes destacan la importancia de las plataformas digitales y redes sociales, pero también de formas más tradicionales de contacto personalizado cara a cara, a través del correo postal o electrónico. Se señala, así mismo, la relevancia de las actividades basadas en las artes, de las herramientas para la comunicación grupal, del uso de estructuras de participación ya existentes (como los consejos municipales de infancia y adolescencia) y la creación de nuevos espacios y grupos de reflexión, think tanks de niños. Según las respuestas recogidas, las actividades ya en proceso y el cuestionamiento de las prácticas vigentes, estarían demostrando las capacidades y visión crítica de los niños, njñas y adolescentes y generando ciertas mejoras en el diseño y difusión de la información, en la forma y contenido de las reglas, medidas y servicios y, en general, en las capacidades organizativas y familiares. Paralelamente, subrayan la importancia de hacer seguimiento de todas las acciones, así como de asegurar la retroalimentación los y las más jóvenes y el compromiso de las personas responsables de la toma de decisiones. Sin embargo, no se aportan ejemplos concretos de que esto se haya producido hasta el momento.

\section{Reflexiones e inquietudes en apoyo de la participación de niños, niñas y adolescentes}

La COVID-19, particularmente las medidas implantadas para hacer frente a la pandemia, han impactado y transformado radicalmente la vida cotidiana de niños, niñas y adolescentes y afectarán y conformarán su cotidianidad

Ver, por ejemplo, para el contexto del Estado Español "Infancia confinada" de Marta Martínez Muñoz, Iván Rodríguez Pascual y Gabriela Velásquez Crespo o "Confiad en los niños” por Lourdes Gaitán, o "Las voces de los niños y de las niñas en situación de confinamiento por el COVID-19, coordinador por Naiara Berasategi Sancho. 
al menos hasta que se desarrolle y extienda una vacuna y/o un tratamiento efectivo frente a la enfermedad. En este contexto, necesitamos reflexionar sobre cómo avanzar en el desarrollo de iniciativas frente a esta crisis sanitaria, económica y social que sean lo más seguras, inclusivas y tengan el mayor impacto posible. Para ello, es fundamental seguir reivindicando la importancia de la inclusión y la participación de los niños, niñas y adolescentes (en particular de aquellos/as en situaciones más vulnerables) en las estructuras y procesos formales de participación comunitaria y pública en todos los ámbitos: en materia de salud pública, en materia educativa, en cuestiones de corresponsabilidad y de uso del espacio urbano y los equipamientos, entre otros. En este sentido, las conclusiones del estudio revelan una serie de debates e inquietudes.

En primer lugar (aunque no han de entenderse en orden de importancia), debemos reflexionar sobre la cuestión del reconocimiento y la reducción de los (pre)juicios basados en la edad; sobre la importancia de celebrar las contribuciones cotidianas de los niños en las respuestas y la configuración de las decisiones públicas. El reconocimiento debe ir acompañado de un compromiso real con la participación infantil, tanto a nivel individual (en cuanto a las decisiones que afecten al niño a nivel particular), como a nivel de influencia colectiva. Sin duda esto se ve favorecido por la existencia y promoción de las oportunidades y espacios (virtuales y no) para que los niños compartan colectivamente sus ideas, pongan de relieve sus preocupaciones, informen y pidan cuentas a los responsables de la toma de decisiones. Un reconocimiento y compromiso real con la participación ante la COVID-19 ha de materializarse también en recursos para los y las profesionales, en particular para aquellos/as en contacto directo con grupos específicos de niños, niñas y adolescentes y comunidades marginadas: recursos temporales (reservar parte de su tiempo) y financiación para las actividades de participación infantil, conocimientos y actualización de sus habilidades digitales y creativas y equipos de protección personal necesarios para llevar a cabo el trabajo.

Otro debate fundamental gira sobre cómo lograr que los procesos participativos sean realmente inclusivos. Para ello cobran gran relevancia los enfoques críticos basados en los derechos, guiados por las preocupaciones de los niños y las comunidades más marginadas, que reclaman revisar las decisiones y medidas en función de las repercusiones que tengan sobre todos los niños, niñas o adolescentes para no dejar a nadie atrás. En un contexto como el que estamos viviendo, esto pasa necesariamente por coordinar las soluciones online y offline. Para ello es fundamental garantizar el suministro eléctrico, el equipamiento digital y el acceso a internet para todos los niños, siempre difundiendo información sobre seguridad en internet y apoyando el aprendizaje entre pares, intercambios de buenas prácticas profesionales seguras e inclusivas. Sin embargo, no debemos confiar y delegar todo en los equipos y materiales digitales, es fundamental garantizar la existencia y el acceso a copias impresas de cualquier material disponible en línea, especialmente cuando se trata de hogares con acceso limitado a la electricidad e internet y para quienes manifiesten una sobrecarga de lo digital.

Esto está directamente vinculado con la promoción del interés superior del niño en la adopción de decisiones económicas, con el desarrollo de leyes y programas para garantizar el acceso inmediato y continuado a los fondos de alimentos, medicamentos, vivienda y servicios esenciales para los niños y personas adultas que sufren pobreza y situaciones vulnerables. Prestaciones de ingreso mínimo vital, especialmente si están diseñadas con una perspectiva de género y de infancia, son fundamentales para que muchos niños, niñas y adolescentes puedan tener acceso a oportunidades, participar y prosperar, de ahí la importancia de la medida recientemente aprobada en España. La esperada recomendación del Consejo de la Unión Europea sobre la garantía infantil y la legislación interna de los Estados miembros y del Consejo de Europa deberían promover acciones que apoyen financieramente a las familias y faciliten el acceso inmediato a servicios de alta calidad.

Como ya se ha señalado en otros trabajos (Larkins et al., 2015), un enfoque de derechos, sustentado en la participación de niños, niñas y adolescentes, nos ofrece orientaciones para revisar las actuales necesidades de acción y de rendición de cuentas en lo que respecta a los efectos de la participación infantil en los servicios y las políticas públicas. Este proceso ha de asentarse sobre una comprensión de las experiencias de quienes se enfrentan a dosis mayores de discriminación y exclusión, para luego entablar diálogos orientados a garantizar el desarrollo efectivo de los derechos. Permitir y fomentar que niños, niñas y adolescentes contribuyan a conformar las respuestas a la COVID-19, comprobar y asegurar que las normas y los servicios desarrollados tienen debidamente en cuenta sus derechos y necesidades, forma parte del cambio necesario para mejorar la seguridad y la eficacia de estas normas y servicios y para promover la salud y el bienestar de la sociedad en su conjunto. Solo así tendremos posibilidades reales de desarrollar soluciones constructivas y a largo plazo ante los problemas y desafíos personales, sanitarios, educativos, comunitarios, económicos y de bienestar social que están surgiendo durante la pandemia y que habrá que continuar atendiendo durante el, previsiblemente, largo periodo de recuperación.

\section{Referencias bibliográficas}

Larkins, C. Ridley, J., Farrelly, N., Austerberry, H., Bilson, A., Hussein, S., Manthorpe, J., Stanley, N. (2015). Children's, Young People's and Parents' Perspectives on Contact: Findings from the Evaluation of Social Work Practices. The British Journal of Social Work, 45(1), 296-312. https://doi.org/10.1093/bjsw/bct135

Warrington, C., Larkins, C. (2019). Children at the centre of safety: challenging the false juxtaposition of protection and participation. Journal of Children's Services, 14 (3),133-142. https://doi.org/10.1108/JCS-09-2019-055 